\title{
Endoskopie an der Grenze zwischen Chirurgie und Gastroenterologie
}

\author{
Gesprächsleiter: Alexander Meining (München) \\ Teilnehmer: $\quad$ Detlef K. Bartsch (Marburg) Ulrike Denzer (Hamburg) \\ Hubertus Feußner (München) Georg Kähler (Mannheim) \\ Thomas Rösch (Hamburg)
}

\begin{abstract}
Frage 1: Das endoskopische Management von chirurgischen Komplikationen (Nahtinsuffizienzen) wird in dieser Ausgabe der Viszeralmedizin von den Kollegen Steinkamp und Wedemeyer näher erläutert. Wie sieht es umgekehrt aus? Wann ist ein chirurgisches Management endoskopischer Komplikationen (vor allem Perforationen) notwendig?
\end{abstract}

Bartsch: Sobald sich nach endoskopischer Perforation eine diffuse Peritonitis oder ein septisches Krankheitsbild entwickelt.

Denzer/Rösch: Im Rahmen der zunehmend komplexen endoskopischen Eingriffe sehen wir vermehrt kleinere endoskopische Perforationen mit kurzfristiger Entzündungsreaktion, die zunächst endoskopisch verschließbar und dann konservativ behandelbar sind. Zudem können akzidentielle Perforationen mittels Vollwandverschluss mit neueren Techniken, z.B. mit dem Ovesco-Clip, in der Regel wirkungsvoll verschlossen werden. Dies gilt für minimalen Luftaustritt ohne nachweisbare Perforation im Mediastinum nach endoskopischer Submukosadissektion (ESD) oder kardianah angelegter transmuraler Zystendrainage sowie für intermittierend freie Luft im Abdomen nach der POEM(perorale endoskopische Myotomie)-Prozedur für Achalasie. Bei einer endoskopisch verschlossenen Perforation sind die frühe Diagnosestellung und der sichere endoskopische Verschluss der Perforation entscheidend; dies bietet ein Zeitfenster für eine konservativ zuwartende Therapie. Zudem sollte man an die Drainage ausgelaufener Flüssigkeit denken, was mittels CT sicher zu diagnostizieren ist. Das Vorgehen sollte möglichst in Absprache mit dem chirurgischen Partner und in regelmäßiger Neubewertung der klinischen Situation erfolgen. Unstrittig ist die Notwendigkeit zur chirurgischen Intervention bei nicht sicher verschlossener und/oder länger symptomatischer Perforation mit klinischer Symptomatik und infizierten Verhalten.
Feußner: Am schwierigsten ist der Entscheid, ob ein endoskopischer Behandlungsversuch überhaupt Erfolg versprechend ist oder nicht besser gleich eine chirurgische Revision erfolgen sollte. Auch heute noch ist die chirurgische Revision der Goldstandard, während das endoskopische Management von iatrogenen endoskopischen Läsionen auch aus gutachterlicher Sicht immer noch besonders gerechtfertigt werden muss. Hier spielen zahlreiche Begleitfaktoren eine Rolle (z.B. Ausmaß der Läsion, Kontaminationsrisiko, spezielle Erfahrung des Endoskopikers usw.).

Wenn man sich für ein primär endoskopisches Management der Komplikation entschlossen hat, muss im weiteren Verlauf die frühzeitige Bereitschaft zum Verfahrenswechsel (d.h. zur chirurgischen Revision) gegeben sein. Aus meiner persönlichen Erfahrung ist es immer wieder erstaunlich, wie wenig interventionelle Gastroenterologen mit dem Phänomen der sekundären Insuffizienz typischerweise nach 5-7 Tagen vertraut sind («Der Teufel kommt am 7. Tag.»). Bis zu diesem Zeitpunkt muss eine ausreichende Überwachung des Patienten gewährleistet sein.

Kähler: Nach wie vor bedürfen Perforationen der Wand des Gastrointestinaltrakts eines sicheren Verschlusses. Während sich bei Perforationen im Rahmen endoskopischer Resektionen (endoskopische Mukosaresektion (EMR) und ESD) die Mehrzahl durch Clips wieder verschließen lässt, muss insbesondere nach Perforationen bei diagnostischer Endoskopie häufig operiert werden. Die konservative Behandlung erfordert für die ersten $48 \mathrm{~h}$ eine engmaschige klinische Überprüfung und gegebenenfalls Korrektur der Entscheidung.

Schwierig ist die Indikationsstellung bei Perforationen an der Papille. Während CT-gesicherte kleine Luftbläschen um das Duodenum herum häufig auftreten, ist in schweren Fällen eine rasche und konsequente operative Sanierung erforderlich. Die verzögerte Versorgung in diesem Bereich hat immer

\section{KARGER}

Fax +497614520714

Information@Karger.de

www.karger.com (c) 2012 S. Karger GmbH, Freiburg

Accessible online at:

www.karger.com/vim
Prof. Dr. Alexander Meining

II. Medizinische Klinik

Klinikum rechts der Isar, Technische Universität München

Ismaninger Straße 22, 81675 München, Deutschland

Alexander.Meining@1rz.tu-muenchen.de 
noch eine schlechte Prognose. Da der Untersucher nach endoskopischen Komplikationen häufig subjektiv befangen ist, sollte die Entscheidung interdisziplinär getroffen werden.

Frage 2: Die endoskopisch gesteuerte Drainage von Abszessen, infizierten Pseudozysten und Nekrosen erscheint aktuell weitgehend etabliert. Wo würden Sie die Grenze der Endoskopie sehen und den Patienten zu einem klassisch chirurgischen Verfahren raten? Gibt es ein endoskopisches Limit bezüglich des Ausmaßes des Befundes? Wie viel Zeit darf/sollte/kann man in etwa beanspruchen, bevor das bisherige Vorgehen eingestellt wird und therapeutisch eskaliert werden sollte?

Bartsch: Es sollte auf ein chirurgisches Verfahren gewechselt werden, wenn ein septisches Krankheitsbild bzw. eine Infektsituation trotz endoskopischer Drainage/Nekrosektomie persistiert oder die Herdausräumung/Entlastung nur unvollständig gelingt. Die Zeit würde ich derzeit immer vom individuellen Befund und Krankheitsverlauf abhängig machen.

Denzer/Rösch: Die endoskopisch gesteuerte Abszessdrainage ist analog der Zystendrainage zu sehen. Hier gibt es eine - nur als Abstract vorliegende - randomisierte kleine Studie aus den USA, die Endoskopie und Chirurgie als gleich effektiv bewertet, bei geringer Hospitalisierungszeit für die Endoskopie. Entsprechend kann die endoskopische Abszessdrainage bzw. die endoskopische Drainage infizierter Pseudozysten bei transluminaler Erreichbarkeit als primäre Therapieoption empfohlen werden. Entscheidend für das Outcome und insbesondere die Vermeidung eines Rezidivs sind unter anderem die suffiziente Zystenspülung initial und gegebenenfalls über eine nasozystische Spüldrainage sowie die ausreichend lange Liegezeit der eingelegten Stents, um die Cystenterostomie offen zu halten.

Für die endoskopische Nekrosektomie gibt es zwei große randomisierte Studien, die zeigen, dass ein «step-up approach» einer offenen chirurgischen Therapie überlegen ist. Eine allerdings sehr kleine, 2012 in JAMA erschienene holländische randomisierte Studie wies die rein endoskopische Vorgehensweise einem «step-up approach» (perkutane Drainage gefolgt von perkutaner Nekrosektomie) ebenfalls als überlegen nach, zumindest was inflammatorische Parameter und Komplikationsrate betrifft. Deswegen empfehlen wir nach Durchführung eines CT primär die endoskopische transmurale Drainage (Endosonographie(EUS)-gesteuert), gegebenenfalls ergänzt durch eine perkutane Drainage bei eingeschränkter endoluminaler Erreichbarkeit und sehr ausgedehnten, sich über das Retroperitoneum verbreitenden Nekrosen. Abhängig vom klinischen Verlauf wird erst bei Versagen dieser Therapien die Operation indiziert.
Feußner: Je foudroyanter der Krankheitsverlauf, umso energischer muss der primäre Behandlungsansatz sein. Wenn beim hochseptischen Patienten die endoskopisch gesteuerte Drainage nicht auf Anhieb, d.h. innerhalb weniger Stunden, zu einer Besserung des Status führt, sollte operiert werden. Je weniger ausgeprägt das Symptombild ist, umso eher gibt es einen Spielraum für endoluminale Behandlungsansätze, sodass eine Step-up-Therapie empfohlen werden kann.

Kähler: Bei gleichen Erfolgsaussichten sollte dem Patienten die endoskopische Behandlung als die am wenigsten invasive Methode primär angeboten werden. Bei deren Versagen (z.B. beim dritten Rezidiv) sollte neu über die OP-Indikation nachgedacht und mit dem Patienten gesprochen werden. Angesichts des bei Patienten mit chronischer Pankreatitis oft erhöhten OP-Risikos kann auch dann noch die endoskopische Drainage die bessere Alternative sein.

Bei speziellen Situationen wie retroperitonealen Nekroseausläufern in die Psoasregion und das kleine Becken sind die Erfolgsaussichten für eine (gegebenenfalls extraperitoneale) Operation besser als bei endoskopischer Ausräumung.

Angesichts der hohen Krankenhausdichte in Deutschland ist es nicht akzeptabel, wenn Indikationen mehr von der Verfügbarkeit der im Haus angewandten Methoden und weniger von den besten Erfolgsaussichten für den Patienten geprägt werden.

Frage 3: NOTES wird in der klinischen Realität entgegen der ursprünglichen Vision des endoskopischen Operierens mit flexiblen Endoskopen über natürliche Körperöffnungen - zwischenzeitlich nahezu ausschließlich als laparoskopisch-endoskopisches Kombinationsverfahren praktiziert. Das eigentliche, von Gastroenterologen entwickelte Konzept wird somit zunehmend eine Domäne des laparoskopisch tätigen MIC-Chirurgen. Worin sehen Sie daher den größten Bedarf, um von «hybrid-NOTES» wieder zum ursprünglichen Konzept zurückzukehren? Welcher Art würden diese Weiterentwicklungen sein bezüglich technischer Änderungen sowie personeller und struktureller Maßnahmen?

Bartsch: Ich sehe im Moment bei den derzeitig verfügbaren Instrumenten/dem Gerätepool keine wirklich sinnvolle reine NOTES-Indikation. Um zum ursprünglichen Konzept zurückzukehren, bedarf es einer Weiterentwicklung der Instrumente, der Endoskope und der sicheren Verschlusstechniken eröffneter Hohlorgane.

Denzer/Rösch: Endoskopische Operationsverfahren wie die POEM oder als Weiterentwicklung die Resektion von submukösen Tumoren in POEM-Technik sind klinische Anwendungen aus dem NOTES-Bereich. Dennoch ist das ursprüngliche 
Konzept des «hybrid-NOTES» aus der Sicht des Endoskopikers kaum umgesetzt. Abhängig von der klinischen Notwendigkeit und den technischen Weiterentwicklungen sind sinnvolle Indikationen zu diskutieren. Erst dann kann über personelle und strukturelle Weiterentwicklungen geredet werden.

Feußner: Grundsätzlich sollte man die Diskussion über «hybrid-NOTES» oder «reine NOTES» zunächst unabhängig vom Bezug zu einer Fachdisziplin sehen. Dass NOTES mit ganz wenigen Ausnahmen bisher in der klinischen Praxis noch nicht praktikabel ist, ist aus meiner Sicht im Wesentlichen drei Faktoren geschuldet:

1. Es fehlen noch immer dedizierte Instrumentensätze und Unterstützungssysteme, die eine sichere Anwendung von NOTES ermöglichen. Erhebliche Defizite bestehen auch bei der Visualisation, in der Sensorik und in der Aktorik. Die für NOTES unbedingt erforderlichen mechatronischen Suppportsyteme sind noch nicht reif für die Praxis.

2. Um erfolgreich NOTES praktizieren zu können, muss der Operateur über domänenspezifisches Wissen/domänenspezifische Erfahrung von zwei Fachdisziplinen verfügen. Konkret bedeutet dies, dass er sowohl über die Fähigkeiten eines erfahrenen interventionellen Gastroenterologen wie auch die eines «sattelfesten» Viszeralchirurgen verfügen muss. Wie sich dieses Problem in Zukunft lösen lassen wird, ist allerdings noch unklar. Vielleicht wird es eines Tages einmal den interdisziplinären NOTES-Interventionalisten geben. Genauso ist aber denkbar, dass NOTESEingriffe in Zukunft grundsätzlich kollegial durch interventionelle Gastroenterologen und Chirurgen gemeinsam durchgeführt werden. Nicht ganz von der Hand zu weisen ist aber auch die Möglichkeit, dass durch intuitive Bedienerschnittstellen und geeignete mechatronische Supportsysteme die operationstechnischen Anforderungen so weit vereinfacht werden können, dass NOTES auch von nur einem durchschnittlich erfahrenen Internisten oder Chirurgen praktiziert werden kann.

3. NOTES für die Praxis setzt auch den Willen und die Fähigkeit zum therapeutischen Paradigmenwechsel voraus. Ein gutes Beispiel dafür ist die Entwicklung der POEM bei der Achalasie. An die Stelle der chirurgischen Kardiomyotomie tritt ein einfaches endo- und transluminales Verfahren, das ohne Weiteres mit dem heute schon zur Verfügung stehenden endoskopischen Instrumentarium erfolgreich durchgeführt werden kann. Es kann mit Sicherheit davon ausgegangen werden, dass auch viele andere Krankheitsbilder potenziell für narbenlose Behandlungstechniken geeignet sind, wenn man den therapeutischen Ansatz verändert (z.B. Ablation statt Resektion).

Kähler: Auch das ursprüngliche Konzept (z.B. NOSCAR White Paper 2005) war ein interdisziplinärer Ansatz, der die etwas artifizielle (und sehr deutsche) Trennung zwischen flexiblen Gastroenterologen und starren Chirurgen auflösen wollte.
Letztlich müssen die Fachgebiete, die die Verantwortung für die Behandlung von spezifischen Krankheitsbildern tragen, entscheiden, ob ihnen NOTES-Konzepte dabei behilflich sind. $\mathrm{Ob}$ die transgastrale Appendektomie sich durchsetzen wird, hängt wohl am ehesten davon ab, ob Chirurgen das als Methode akzeptieren. Gastroenterologen müssen entscheiden, ob NOTES-Konzepte, beispielsweise als transgastrale Laparoskopie anstelle der diagnostischen (transabdominellen) Laparoskopie oder zum Tumorstaging, gegebenenfalls auch zur lokalen Tumortherapie sinnvoll eingesetzt werden können.

Hilfreich wären einsatzfähige flexible Operationsendoskope. Deren Entwicklung setzt voraus, dass die medizintechnische Industrie an einen profitablen Einsatz in absehbarer Zukunft glaubt. Wie bei anderen trainingsbedürftigen Interventionen wäre die Etablierung des Berufsbildes eines Endoskopiespezialisten förderlich.

Frage 4: Das Problem einer alleinigen endoskopischen Therapie von frühen Neoplasien besteht vor allem in der fehlenden Möglichkeit einer Lymphknotendissektion. Nehmen wir an, Sie betreuen einen 30-jährigen Patienten mit der Zufallsdiagnose eines gut differenzierten mukosalen Karzinoms (anhand einer Biopsie festgestellt) am gastroösophagealen Übergang. Die Läsion ist leicht erhaben und hat eine Ausdehnung von ca. 15 mm. Welche weitere Diagnostik erachten Sie für notwendig? Wann würden Sie von einer alleinigen endoskopischen Therapie absehen?

Bartsch: EUS. Bei suspekten Lymphknoten oder >uT1.

Denzer/Rösch: Die Komponente eines jugendlichen Tumorpatienten ist vorwiegend emotional besetzt (auch von Ärzten) und wenig durch vergleichende Daten mit durchschnittlich oder besonders alten Tumorpatienten zu belegen. Bei einem so jungen Patienten sollte sicherlich nach Tumorsyndromen und genetischen Erkrankungen gefahndet werden. Bei einem gut differenzierten mukosalen Adenokarzinom am gastroösophagealen Übergang würden wir zunächst eine erneute endoskopische Beurteilung unter Verwendung einer Aufsatzkappe und Färbung der Mukosa vornehmen. Wichtig ist eine genaue Lokalisationsdiagnostik hinsichtlich einer geplanten endoskopischen Abtragung mittels fokaler und longitudinaler Biopsien. Eine EUS kann die Diagnostik zur Beurteilung der Lymphknotenstationen und der Ösophaguswandschichtung komplettieren; die Treffsicherheit in der Beurteilung der lokalen Ausdehnung des Tumors und von perimurealen Lymphknoten ist aber begrenzt. Erscheint der Befund resektabel, wäre der nächste Schritt die endoskopische Resektion mit histologischer Aufarbeitung des Resektats. Bei Erfüllen der Kriterien ist eine engmaschige Nachsorge vermutlich ausreichend. Bei Submukosainfiltration bedingt das junge Patientenalter derzeit eine chirurgische Nachresektion mit 
Lymphknotendissektion; bei entsprechender endoskopischer En-bloc- und R0-Resektion eines Cardiatumors ohne Barrett ist die Nachresektion gegenüber einer reinen (laparoskopischen) Lymphknotenentfernung ebenso wenig durch Daten abgesichert und bleibt eine individuelle Entscheidung. Bei älteren Patienten mit Vorerkrankungen und eingeschränkter OP-Fähigkeit wird wohl im Allgemeinen bei endoskopischer R0-Resektion auch eine alleinige laparoskopische Lymphknotenresektion diskutiert werden, wobei diesbezüglich Daten fehlen.

Feußner: Der hier geschilderte Befund legt natürlich primär die endoskopische Exzision nahe, wenn in der EUS ein T1m-Stadium und Lymphknotennegativität nachgewiesen ist. Wenn dabei anhand eines onkologisch einwandfreien Resektats (kein Piecemeal-Präparat!) eine R0-Resektion bei gut differenziertem Karzinom bewiesen werden kann, muss das weitere Management mit dem Patienten besprochen werden. Die Wahrscheinlichkeit einer Lymphknotenmetastasierung ist unter diesen Umständen zwar sehr gering, aber keineswegs gleich null. Andererseits ist die Letalität eines chirurgischen Eingriffs bei einem 30-jährigen, ansonsten gesunden Patienten in erfahrenen Zentren zwar auch äußerst gering, aber das Risiko, bei einem unter Umständen gar nicht mehr notwendigen Eingriff zu versterben, ist ebenfalls nicht nur rein theoretisch. Eine Einbindung des Patienten in diese Entscheidung ist unbedingt erforderlich. In dubio ist auch heute noch die chirurgische Resektion das Standardvorgehen.

Kähler: Solche Fälle besprechen wir ausnahmslos im Interdisziplinären Tumorboard. Im skizzierten Fall würden wir endosonographieren und eventuell ein CT machen. Falls nodal in beiden Modalitäten negativ, würden wir eine ESD anschlieBen. Falls $\mathrm{G}>2, \mathrm{~L}>0, \mathrm{~N}>0, \mathrm{Pn}>0$ und $\mathrm{T}>\mathrm{T} 1 \mathrm{sm} 1$ würden wir eine OP dringend empfehlen. Falls nur eine mukosale Ausbreitung vorliegt, empfehlen wir Beobachtung, bei sm1 ausführliche Aufklärung und Patientenentscheidung - im vorliegenden Fall mit Beratung zur OP.

Frage 5: Die interventionelle Endoskopie löst zunehmend traditionell chirurgische Verfahren ab. Dementsprechend steigt aber auch deren Komplexität. Besondere Kenntnisse und Fähigkeiten werden daher impliziert. Welche Voraussetzungen sollten daher gegeben sein, bevor komplexe endoskopische Eingriffe durchgeführt werden? Bedarf es hierzu einer einheitlichen Regelung, gegebenenfalls auch bezüglich der festgeschriebenen Fort- und Weiterbildungsinhalte?

Denzer/Rösch: Das Erlernen und die Ausübung komplexer endoskopischer Operationstechniken bedingt eine fundierte praktische Ausbildung, eingebettet in die Inhalte der gastroenterologischen Weiterbildung. Die Teilnahme an speziellen
Kursen mit Trainingsmodellen ersetzt dabei nicht die praktische Lernkurve, kann aber Grundlagen zu Theorie und praktischer Technik vermitteln. Die Aufstellung spezieller zusätzlicher Fort- und Weiterbildungsverpflichtungen erachten wir dabei nicht als unbedingte Voraussetzung. Vielmehr kann zusätzlich ein Ausbildungsaustausch in Form von Rotationen bzw. Hospitationen an spezialisierten Zentren sinnvoll sein. Komplexe und vor allem innovative Verfahren sollten auf Zentren mit interdisziplinärer Expertise und der Fähigkeit sowie dem Willen zur kritischen begleitenden Evaluation beschränkt sein.

Feußner: Einheitliche Regelungen entspringen häufig dem übersteigerten Qualitätsmanagementeifer und sind tendenziell eine Fortschrittsbremse. Damit soll jedoch keineswegs einer «see one, do one, teach one»-Mentalität das Wort geredet werden. In dafür geeigneten Institutionen wie den Universitätsklinika oder spezialisierten Zentren müssen unter transparenten, wissenschaftlich korrekten Rahmenbedingungen innovative Therapieansätze möglich sein, die auch in den traditionellen Regelwerken (Fort- und Weiterbildungsinhalte) noch nicht beschrieben sind.

Kähler: Gerade bei der Etablierung neuer Verfahren können nicht alle Eventualitäten reglementiert werden. Wünschenswert ist eine bedarfsorientierte Spezifizierung von endoskopischen Qualifikationen.

- So ist für die Mehrheit der Gastroenterologen, die sich niederlassen wollen, die Qualifikation für die endoskopisch retrograde Cholangiopankreatikographie (ERCP) nicht erforderlich; stattdessen sollte es eine Art qualitätsgesicherte Fachkunde ERCP für klinisch tätige Ärzte geben.

- Ähnliche Regelungen wären sinnvoll für die EUS und die ESD.

- Einzelne Fach- und Spezialgebiete haben besonderes Interesse an einzelnen Untersuchungsmethoden (Bronchoskopie für die Pulmologie und Thoraxchirurgie, Koloskopie in der Koloproktologie, rektale EUS für die Onkochirurgie des Rektums usw.). Daher wäre es qualitätssichernd, Zertifikate oder Qualifikationen für einzelne Untersuchungsarten von verschiedenen Fach- und Teilgebieten aus erwerben zu können.

- Da sich das Spektrum der Methoden ständig weiterentwickelt, sind letztlich einzelne Abteilungen und deren Leitungen als Endoskopie-spezifische Strukturen erforderlich. Diese sollten Endoskopie schon aus versorgungspolitischen Gründen nicht exklusiv wahrnehmen, aber auf die Entwicklung, Implementierung und wissenschaftliche Prüfung sowie die Ausbildung fokussiert sein. Dafür ist einerseits eine Einbindung in übergeordnete klinische Strukturen erforderlich, um in die Fachgebiete integriert zu bleiben. Andererseits ist eine relative finanzielle und administrative Autonomie erforderlich, um die Erfordernisse einer stabilen Entwicklung zu sichern. 


\section{Teilnehmer}

Prof. Dr. med. Detlef K. Bartsch

Klinik für Visceral-, Thorax- und Gefäßchirurgie

Philipps-Universität Marburg

Baldingerstraße, 35037 Marburg, Deutschland

bartsch@med.uni-marburg.de

PD Dr. Ulrike Denzer

Klinik für Interdisziplinäre Endoskopie

Universitätsklinik Hamburg-Eppendorf

Martinistraße 52, 20246 Hamburg, Deutschland

u.denzer@uke.de

Prof. Dr. Hubertus Feußner

Chirurgische Klinik und Poliklinik

Klinikum rechts der Isar, Technische Universität München

Ismaninger Straße 22, 81675 München, Deutschland

hubertus.feussner@tum.de
Prof. Dr. med. Georg Kähler

Sektion Endoskopie, Chirurgische Universitätsklinik Mannheim Medizinische Fakultät Mannheim

Theodor-Kutzer-Ufer 1-3, 68167 Mannheim, Deutschland

Georg.Kaehler@umm.de

Prof. Dr. med. Thomas Rösch

Klinik für Interdisziplinäre Endoskopie

Universitätsklinikum Hamburg-Eppendorf

Martinistraße 52, 20246 Hamburg, Deutschland

t.roesch@uke.de 